\title{
Association of IL-4 promoter polymorphisms with asthma: a meta-analysis
}

\author{
L. Tang ${ }^{1}$, H.G. Lin ${ }^{2}$ and B.F. Chen ${ }^{2}$ \\ ${ }^{1}$ School of Basic Medical Science, Changsha Medical University, \\ Changsha, China \\ ${ }^{2}$ School of Biomedical Sciences, Faculty of Medicine, \\ The Chinese University of Hong Kong, Hong Kong SAR, China \\ Corresponding author: B.F. Chen \\ E-mail: cbifeng@gmail.com
}

Genet. Mol. Res. 13 (1): 1383-1394 (2014)

Received January 17, 2013

Accepted July 23, 2013

Published February 28, 2014

DOI http://dx.doi.org/10.4238/2014.February.28.11

\begin{abstract}
This study aimed to more precisely assess the correlation between interleukin-4 (IL-4) promoter polymorphisms and the susceptibility risk of asthma. We conducted association studies on IL-4 promoter C-33T, C-589T, and G-1098T polymorphisms with asthma using data obtained from MEDLINE up to September 2011. Results showed that the polymorphisms IL-4 C-33T and C-589T were significantly associated with asthma; however, significant associations were found only in the European population. In addition, the TT $+\mathrm{CT}$ genotype was significantly associated with asthma in adults and no significant association was found in asthma status subgroup analyses. This meta-analysis showed that IL-4 C-589T and C-33T were associated with asthma in Europeans. To further confirm correlations between polymorphisms of the IL-4 promoter with asthma susceptibility, studies involving a larger number of patients worldwide are necessary.
\end{abstract}

Key words: IL-4; Promoter; Polymorphisms; Meta-analysis; Asthma 


\section{INTRODUCTION}

Asthma is a complex inflammatory disease caused by a variety of stimuli, which is characterized by airway responsiveness, reversible airway obstruction, and inflammation. A multitude of cytokines is important in this inflammation (Zhu et al., 2000). A significant role for cytokines in the pathogenesis of asthma is evident, as they have been shown to play a critical role in immunoglobulin E (IgE) synthesis, eosinophil recruitment, and bronchial hyperresponsiveness (Renauld, 2001).

The Th2 cytokines [interleukin (IL)-4, IL-5, IL-9, IL-10, and IL-13] have a substantial effect on the pathogenesis of atopic diseases. IL-4 is produced by activated T cells, mast cells, and basophils, which participate in IgE synthesis and Th2 phenotype differentiation in T cells, and play a crucial role in the development of atopic diseases including asthma (Beghe et al., 2003).

Genome-wide association studies have suggested that an asthma susceptible locus could be mapped to chromosomes 5q31-33, covering genes encoding for human IL-4, IL-13, and IL-16. Moreover, the IL-4 gene was associated with asthma in more than three independent study populations (Liu et al., 2003; Michel et al., 2010). A polymorphism (C-589T) in the IL-4 promoter region seems to be related to elevated serum levels of IgE (Berenguer et al., 2012). Another single nucleotide polymorphism (SNP), C-33T, located in the 5'-untranslated region (UTR) of IL-4, was reported to be associated with asthma (Kim et al., 2010). Additionally, although a third polymorphism, G-1098T, has been investigated in several cohorts, its correlation with asthma varies across studies (Gervaziev et al., 2006; Amirzargar et al., 2009; Baye et al., 2011). Further investigations indicated that the polymorphisms C-589T and C-33T were associated with asthma by some authors, but by no means all (Elliott et al., 2001; Haller et al., 2009), which may be due to small sample sizes, low statistical power, and/or clinical heterogeneity.

Due to these conflicting and controversial results, combined evidence needs to be taken into account in order to assess the association between IL-4 promoter polymorphisms and asthma. In the present study, we carried out a meta-analysis to evaluate the association of IL-4 promoter polymorphisms with asthma susceptibility risk among different ethnic and asthma status groups.

\section{MATERIAL AND METHODS}

\section{Identification and selection of relevant studies}

The key words ("Interleukin-4" or "IL-4") and ("asthma") were used in the PUBMED database to identify relevant publications. Meanwhile, additional studies were identified by cross-referencing within original or review articles. Studies were not restricted to any particular language. Data from any fully published paper, excluding meeting or conference abstracts, were extracted. A study was included in the current meta-analysis if it conformed to the following criteria: i) an unrelated case-control design was used; ii) allele or genotype frequencies were available; and iii) frequencies of SNPs were in Hardy-Weinberg (HW) equilibrium.

\section{Data extraction}

Information of first author, year of publication, type of study design, ethnicity, age, 
asthma status, total sample size, number of cases and controls, genotype, haplotypes containing SNPs C-33T and C-589T, and allele frequencies were collected from each study. Two independent authors (L. Tang and H-G. Lin) extracted all data from each study and consensus was achieved for all studies.

\section{Statistical methods}

The odds ratio (OR) and the $95 \%$ confidence interval $(95 \% \mathrm{CI})$ were calculated for each study. Variation and heterogeneity were evaluated using Cochran's Q-statistic. If significant heterogeneity was observed across studies $(\mathrm{P}<0.10)$, the random-effect model was used for meta-analysis, otherwise, the fixed-effect model was used. The effect of heterogeneity was also measured by the $\mathrm{I}^{2}$ value: $\mathrm{I}^{2}=100 \% \mathrm{x}(\mathrm{Q}$ - d.f.) / Q. Calculation of power was obtained at the 0.05 significance level, assuming an OR of 1.5 (small effect size). G*Power was used to perform the power analysis (http://www.psycho.uni-duesseldorf.de/aap/projects/gpower).

\section{Publication bias}

Publication bias was assessed using the Egger test. A P value $<0.05$ was considered to indicate statistically significant publication bias. Sensitivity analysis excluding individual studies was performed in the meta-analysis. All statistical analyses were conducted with RevMan 5 (Oxford, UK) and STATA10.0 (http://www.stata.com) programs.

\section{RESULTS}

\section{Studies included in the meta-analysis}

There were 160 papers identified through the initial search. After browsing titles and abstracts, 14 reviews were excluded, 19 articles were excluded because they were not conducted with human subjects, and 8 articles were excluded because they did not explore associations with asthma. After reading the full articles, 101 studies were excluded for not exploring IL-4 genetic polymorphisms, leaving 18 studies for full publication review (Rosenwasser et al., 1995; Laitinen et al., 1997; Sandford et al., 2000; Zhu et al., 2000; Noguchi et al., 1998, 2001; Freidin et al., 2003; Basehore et al., 2004; Park et al., 2004; Donfack et al., 2005; Gervaziev et al., 2006; Amirzargar et al., 2009; Wang et al., 2009; Beghe et al., 2003, 2010; Yang et al., 2011; Baye et al., 2011; Dmitrieva-Zdorova et al., 2012). Seven studies were excluded because they were family-based (Rosenwasser et al., 1995; Laitinen et al., 1997; Zhu et al., 2000; Noguchi et al., 1998, 2001; Beghe et al., 2003; Freidin et al., 2003). Four studies were excluded because allele frequencies were in HW disequilibrium (Gervaziev et al., 2006; Amirzargar et al., 2009; Wang et al., 2009; Dmitrieva-Zdorova et al., 2012). Meanwhile, 14 studies were added by cross-referencing (Walley and Cookson, 1996; Takabayashi et al., 2000; Suzuki et al., 2000; Hijazi and Haider, 2000; Cui et al., 2003a,b; Lee et al., 2004; Zhang et al., 2005; Isidoro-García et al., 2005; Chiang et al., 2007; Kamali-Sarvestani et al., 2007; Hosseini-Farahabadi et al., 2007; Mak et al., 2007; Rad et al., 2010). Therefore, a total of 21 studies were included in the meta-analysis (Table 1). Overall, eligible studies involved a total of 6421 asthma patients and 4755 healthy controls from 7 European, 14 Asian, and 3 African- 
American sample populations. Four studies comprised two or three different subpopulations (Basehore et al., 2004; Donfack et al., 2005; Zhang et al., 2005; Baye et al., 2011). Each population was treated independently. Therefore, 30 separate comparisons were possible. The meta-analysis was performed if there were at least two comparisons; therefore, a total of 29 comparisons were performed in this meta-analysis.

\begin{tabular}{|c|c|c|c|c|c|c|c|c|c|}
\hline \multirow[t]{2}{*}{ SNP } & \multirow{2}{*}{$\begin{array}{l}\text { Reference } \\
\text { (1st author) }\end{array}$} & \multirow[t]{2}{*}{ Country (ethnic) } & \multirow[t]{2}{*}{ Year } & \multirow[t]{2}{*}{ Subjects age } & \multirow[t]{2}{*}{ Asthma status } & \multicolumn{2}{|c|}{ Number } & \multirow{2}{*}{ OR, P value } & \multirow{2}{*}{$\begin{array}{c}\text { Power }^{\mathrm{a}} \\
(\alpha=0.05, \mathrm{OR}=1.5)\end{array}$} \\
\hline & & & & & & Case & Control & & \\
\hline \multirow[t]{24}{*}{ C-589T } & \multirow[t]{3}{*}{ Basehore } & USA & 2004 & Adult & $\mathrm{A}$ & 233 & 245 & $1.04,0.82$ & 70.4 \\
\hline & & African-American & 2004 & Adult & A & 168 & 269 & $0.88,0.37$ & 67.1 \\
\hline & & Hispanic & 2004 & Adult & $\mathrm{A}$ & 116 & 130 & $1.18,0.38$ & 46.8 \\
\hline & Park & Korean & 2004 & Adult & $\mathrm{A} / \mathrm{NA}$ & 532 & 170 & $0.94,0.76$ & 84.2 \\
\hline & Beghe & UK & 2009 & Adult & A & 299 & 176 & $1.01,0.99$ & 70.2 \\
\hline & Sandford & New Zealand & 2000 & Adult & $\mathrm{A}$ & 233 & 143 & $1.39,0.097$ & 61.4 \\
\hline & \multirow[t]{2}{*}{ Baye } & Caucasian & 2011 & Children & $\mathrm{A} / \mathrm{NA}$ & 413 & 298 & $2.00,0.00002$ & 84.6 \\
\hline & & African-American & 2011 & Children & $\mathrm{A} / \mathrm{NA}$ & 315 & 51 & $0.56,0.008$ & 60.8 \\
\hline & \multirow[t]{2}{*}{ Donfack } & European & 2005 & Adult & $\mathrm{A} / \mathrm{NA}$ & 126 & 205 & $0.98,0.92$ & 57.1 \\
\hline & & African-American & 2005 & Adult & $\mathrm{A} / \mathrm{NA}$ & 205 & 183 & $1.17,0.29$ & 62.9 \\
\hline & Chiang & Taipei & 2007 & Adult & Unknown & 167 & 112 & $4.06,<0.0001$ & 51.2 \\
\hline & Kamali-Sarvestani & Iran & 2007 & Adult & A & 203 & 112 & $2.05,0.007$ & 55.3 \\
\hline & Takabayashi & Japanese & 2000 & Children & A & 100 & 100 & $4.11,<0.0001$ & 40.9 \\
\hline & Lee & Korean & 2004 & Children & $\mathrm{A} / \mathrm{NA}$ & 256 & 100 & $0.88,0.57$ & 59.8 \\
\hline & $\mathrm{Rad}$ & Iran & 2010 & Adult & Unknown & 64 & 65 & $0.76,0.42$ & 30.5 \\
\hline & Cui & Chinese & 2003 & Adult & A & 98 & 103 & $0.76,0.27$ & 41.1 \\
\hline & Hijazi & Kuwaiti Arabs & 2000 & Adult & $\mathrm{A} / \mathrm{NA}$ & 84 & 100 & $1.23,0.4$ & 38.7 \\
\hline & Walley & UK & 1996 & Adult & A & 124 & 59 & $1.34,0.25$ & 38.6 \\
\hline & Cui & Chinese & 2003 & Adult & A & 241 & 175 & $0.84,0.29$ & 65.6 \\
\hline & Mak & Chinese & 2007 & Adult & $\mathrm{A} / \mathrm{NA}$ & 292 & 292 & $0.94,0.67$ & 77.9 \\
\hline & Hosseini & Iran & 2007 & Adult & A & 30 & 50 & $3.14,0.005$ & 22.4 \\
\hline & \multirow[t]{3}{*}{ Zhang } & Chinese & 2005 & Adult & $\mathrm{A} / \mathrm{NA}$ & 145 & 156 & $0.83,0.40$ & 53.4 \\
\hline & & Malayan & 2005 & Adult & $\mathrm{A} / \mathrm{NA}$ & 73 & 93 & $1.09,0.72$ & 35.9 \\
\hline & & Hindoo & 2005 & Adult & $\mathrm{A} / \mathrm{NA}$ & 85 & 99 & $1.19,0.49$ & 38.5 \\
\hline \multirow[t]{7}{*}{ C-33T } & Yang & Chinese & 2011 & Adult & A/NA & 202 & 205 & $0.99,0.713$ & 64.8 \\
\hline & \multirow{3}{*}{ Basehore } & USA & 2004 & Adult & A & 233 & 245 & $1.55,0.014$ & 70.9 \\
\hline & & African-American & 2004 & Adult & A & 168 & 269 & $1.04,0.79$ & 67.5 \\
\hline & & Hispanic & 2004 & Adult & $\mathrm{A}$ & 116 & 130 & $1.19,0.37$ & 47.1 \\
\hline & Park & Korean & 2004 & Adult & $\mathrm{A} / \mathrm{NA}$ & 532 & 170 & $0.89,0.44$ & 84.5 \\
\hline & Isidoro-García & Caucasian & 2005 & Adult & $\mathrm{A} / \mathrm{NA}$ & 133 & 79 & $1.82,0.07$ & 41.2 \\
\hline & Suzuki & Japanese & 2000 & Adult & A & 120 & 120 & $1.02,0.92$ & 46.3 \\
\hline G-1098T & Baye & African-American & 2011 & Children & $\mathrm{A} / \mathrm{NA}$ & 315 & 51 & $0.47,0.008$ & 60.8 \\
\hline
\end{tabular}

$\mathrm{OR}=$ odds ratio; $\mathrm{a}=$ power calculations assume $\alpha=0.05$ and small effect size $(0.1)$ or $\mathrm{OR}=1.5 ; \mathrm{SNP}=$ single nucleotide polymorphism; $\mathrm{A}=$ atopic; $\mathrm{NA}=$ nonatopic.

Two IL-4 gene polymorphisms were investigated in the meta-analysis. The distribution of genotypes was in HW equilibrium in these studies $(\mathrm{P}<0.05)$. Ethnicity-specific metaanalyses were conducted for European, Asian, and African-American populations. Subgroup analyses were also performed with respect to asthma status.

\section{Main results}

Results of the meta-analysis of the relationship between IL-4 promoter polymorphisms and asthma are shown in Table 2, Figures 1 and 2. Due to an insufficient number of studies, the meta-analysis of G-1098T haplotypes containing SNPs C-33T and C-589T with asthma 
was not conducted in this study. In this analysis, the overall ORs for IL-4 C-33T were significantly increased in asthma $(\mathrm{OR}=1.14,95 \% \mathrm{CI}=1.00-1.31, \mathrm{P}=0.04)$. Moreover, a significant association was revealed in Europeans $(\mathrm{OR}=1.26,95 \% \mathrm{CI}=1.03-1.55, \mathrm{P}=0.02)$, but not in Asians $(\mathrm{OR}=1.03,95 \% \mathrm{CI}=0.85-1.26, \mathrm{P}=0.74)$. Neither the TT nor the TT $+\mathrm{CT}$ genotype of $\mathrm{C}-33 \mathrm{~T}$ were associated with asthma $(\mathrm{P}>0.05)$. A significant association was found between $\mathrm{C}-589 \mathrm{~T}$ and asthma $(\mathrm{OR}=1.20,95 \% \mathrm{CI}=1.01-1.42, \mathrm{P}=0.04)$. However, the ethnic-specific meta-analysis showed that this association only existed in Europeans $(\mathrm{OR}=1.27,95 \% \mathrm{CI}=$ $1.10-1.46, \mathrm{P}=0.0009)$. The meta-analysis showed a significant association of the $\mathrm{TT}+\mathrm{CT}$ genotype of $\mathrm{C}-589 \mathrm{~T}$ with asthma $(\mathrm{OR}=1.30,95 \% \mathrm{CI}=1.11-1.53, \mathrm{P}=0.001)$ but not the TT genotype $(\mathrm{OR}=1.11,95 \% \mathrm{CI}=0.86-1.44, \mathrm{P}=0.43)$. With respect to these genotypes, results based on ethnicity showed no difference from the combined results.

\begin{tabular}{|c|c|c|c|c|c|c|c|c|c|c|c|c|}
\hline \multirow[t]{2}{*}{ SNP } & \multirow[t]{2}{*}{ Comparison } & \multicolumn{2}{|c|}{ Sample size } & \multirow{2}{*}{$\begin{array}{l}\text { No. of } \\
\text { studies }\end{array}$} & \multicolumn{3}{|c|}{ Test of association } & \multirow[t]{2}{*}{ Model } & \multicolumn{3}{|c|}{ Test of heterogeneity } & \multirow{2}{*}{$\begin{array}{l}\text { Publication bias } \\
\text { P value } \\
\text { (Egger's test) }\end{array}$} \\
\hline & & Disease & Control & & OR & $95 \% \mathrm{CI}$ & P value & & Q & P value & $\mathrm{I}^{2}$ & \\
\hline \multirow[t]{11}{*}{ C-589T } & $T v s C$ & & & & & & & & & & & \\
\hline & European & 3088 & 2502 & 7 & 1.24 & $1.03-1.50$ & 0.02 & $\mathrm{~F}$ & 10.3 & 0.11 & 42 & 0.41 \\
\hline & Asian & 4740 & 3454 & 14 & 1.31 & $0.99-1.74$ & 0.06 & $\mathrm{R}$ & 72 & $<0.00001$ & 82 & 0.11 \\
\hline & African-American & 1376 & 1066 & 3 & 0.85 & $0.58-1.25$ & 0.42 & $\mathrm{R}$ & 8 & 0.02 & 75 & 0.14 \\
\hline & $\begin{array}{l}\text { Overall } \\
T T \text { v } T C+C C\end{array}$ & 9204 & 6962 & 24 & 1.20 & $1.01-1.42$ & 0.04 & $\mathrm{R}$ & 100 & $<0.00001$ & 77 & 0.47 \\
\hline & Overall & 2312 & 2174 & 15 & 1.11 & $0.86-1.44$ & 0.43 & $\mathrm{R}$ & 24.6 & 0.001 & 60 & 0.10 \\
\hline & European & 269 & 438 & 2 & 0.90 & $0.17-4.86$ & 0.91 & $\mathrm{R}$ & 3.1 & 0.08 & 68 & NA \\
\hline & $\begin{array}{l}\text { Asian } \\
T T+T C \text { v } C C\end{array}$ & 1838 & 1553 & 12 & 1.11 & $0.82-1.49$ & 0.51 & $\mathrm{R}$ & 31.4 & 0.0009 & 65 & 0.09 \\
\hline & Overall & 2020 & 1798 & 17 & 1.30 & $1.11-1.53$ & 0.001 & $\mathrm{~F}$ & 16.3 & 0.43 & 2 & 0.07 \\
\hline & European & 602 & 749 & 3 & 1.29 & $1.03-1.62$ & 0.03 & $\mathrm{~F}$ & 2 & 0.89 & 0 & 0.10 \\
\hline & Asian & 1838 & 1553 & 13 & 1.36 & $1.07-1.72$ & 0.01 & F & 15.8 & 0.20 & 24 & 0.97 \\
\hline \multirow[t]{10}{*}{ C-33T } & $T v s C$ & & & & & & & & & & & \\
\hline & European & 1068 & 1186 & 3 & 1.26 & $1.03-1.55$ & 0.02 & F & 4.6 & 0.10 & 56 & 0.55 \\
\hline & Asian & 1708 & 990 & 3 & 1.03 & $0.85-1.26$ & 0.74 & $\mathrm{~F}$ & 7.4 & 0.73 & 0 & 0.64 \\
\hline & $\begin{array}{l}\text { Overall } \\
T T \text { vs } T C+C C\end{array}$ & 3008 & 2436 & 7 & 1.14 & $1.00-1.31$ & 0.04 & $\mathrm{~F}$ & 8.7 & 0.31 & 15 & 0.08 \\
\hline & Overall & 455 & 404 & 3 & 1.07 & $0.78-1.47$ & 0.66 & $\mathrm{~F}$ & 2 & 0.95 & 0 & NA \\
\hline & European & 133 & 79 & 1 & 1.82 & $0.07-44.72$ & 0.72 & - & & - & - & - \\
\hline & $\begin{array}{l}\text { Asian } \\
T T+T C \text { vs } C C\end{array}$ & 322 & 325 & 2 & 1.07 & $0.78-1.47$ & 0.69 & $\mathrm{~F}$ & 1 & 0.99 & 0 & NA \\
\hline & Overall & 455 & 404 & 3 & 1.08 & $0.69-1.69$ & 0.75 & $\mathrm{R}$ & 5.6 & 0.06 & 64 & 0.08 \\
\hline & European & 133 & 79 & 1 & 1.84 & $0.94-3.60$ & 0.08 & - & & - & & - \\
\hline & Asian & 322 & 325 & 2 & 0.66 & $0.35-1.24$ & 0.20 & $\mathrm{~F}$ & 1 & 0.33 & 0 & NA \\
\hline
\end{tabular}

$\mathrm{OR}=$ odds ratio $; 95 \% \mathrm{CI}=95 \%$ confidence interval; $\mathrm{NA}=$ not available; $\mathrm{F}=$ fixed-effect model; $\mathrm{R}=$ random-effect model.

Subgroups were also analyzed based on asthma status: unknown (atopy status nondefined); atopic asthma; and nonatopic asthma. In the atopic and nonatopic asthma subgroups, no associations between C-589T and asthma were revealed, except for TT vs TC+CC in the asthma group. Furthermore, no association was discovered between $\mathrm{C}-33 \mathrm{~T}$ and asthma (Figures 3 and 4). 


\begin{tabular}{|c|c|c|c|c|c|c|c|c|c|c|c|}
\hline Studv or Subgroup & $\begin{array}{l}\text { case } \\
\text { Events }\end{array}$ & Total & $\begin{array}{l}\text { Contr } \\
\text { Events }\end{array}$ & $\begin{array}{l}\text { ol } \\
\text { Total }\end{array}$ & Weight & $\begin{array}{c}\text { Odds Ratio } \\
\text { M-H, Random, } 95 \% \mathrm{Cl}\end{array}$ & Year & & $\begin{array}{r}\text { Odds } \mathrm{F} \\
\text { M-H, Rando }\end{array}$ & $\begin{array}{l}\text { Ratio } \\
\text { lom. } 95 \% \mathrm{Cl}\end{array}$ & \\
\hline Walley 1996 & 82 & 248 & 29 & 108 & $3.9 \%$ & $1.35[0.82,2.22]$ & 1996 & & & + & \\
\hline Hijazi 2000 & 133 & 168 & 151 & 200 & $3.9 \%$ & $1.23[0.75,2.02]$ & 2000 & & & - & \\
\hline Takabayashi 2000 & 188 & 200 & 141 & 200 & $3.1 \%$ & $6.56[3.40,12.66]$ & 2000 & & & & \\
\hline Sandford 2000 & 96 & 466 & 45 & 286 & $4.4 \%$ & $1.39[0.94,2.05]$ & 2000 & & & - & \\
\hline Cui 2003 & 371 & 482 & 280 & 350 & $4.7 \%$ & $0.84[0.60,1.17]$ & 2003 & & & & \\
\hline Cui 2003 & 149 & 196 & 166 & 206 & $4.0 \%$ & $0.76[0.47,1.23]$ & 2003 & & & & \\
\hline Lee 2004 & 413 & 512 & 165 & 200 & $4.2 \%$ & $0.88[0.58,1.35]$ & 2004 & & & & \\
\hline Park 2004 & 862 & 1064 & 272 & 340 & $4.8 \%$ & $1.07[0.79,1.45]$ & 2004 & & & - & \\
\hline Basehore-2 2004 & 215 & 336 & 360 & 538 & $4.9 \%$ & $0.88[0.66,1.17]$ & 2004 & & & & \\
\hline Basehore-3 2004 & 90 & 232 & 91 & 260 & $4.5 \%$ & $1.18[0.82,1.70]$ & 2004 & & & - & \\
\hline Basehore-1 2004 & 68 & 466 & 69 & 490 & $4.6 \%$ & $1.04[0.73,1.50]$ & 2004 & & & - & \\
\hline Zhang -1 2005 & 236 & 290 & 262 & 312 & $4.3 \%$ & $0.83[0.55,1.27]$ & 2005 & & & & \\
\hline Zhang-2 2005 & 90 & 146 & 116 & 186 & $4.1 \%$ & $0.97[0.62,1.52]$ & 2005 & & & - & \\
\hline Donfack-1 2005 & 49 & 252 & 81 & 410 & $4.4 \%$ & $0.98[0.66,1.46]$ & 2005 & & & - & \\
\hline Donfack-2 2005 & 278 & 410 & 235 & 366 & $4.9 \%$ & $1.17[0.87,1.58]$ & 2005 & & & - & \\
\hline Zhang-3 2005 & 43 & 170 & 44 & 198 & $4.0 \%$ & $1.19[0.73,1.92]$ & 2005 & & & & \\
\hline Mak 2007 & 453 & 584 & 459 & 584 & $5.0 \%$ & $0.94[0.71,1.24]$ & 2007 & & & & \\
\hline Hosseini-Farahabadi 2007 & 18 & 60 & 12 & 100 & $2.5 \%$ & $3.14[1.39,7.12]$ & 2007 & & & & \\
\hline Chi ang 2007 & 313 & 334 & 176 & 224 & $3.6 \%$ & $4.06[2.36,7.01]$ & 2007 & & & $\rightarrow$ & \\
\hline Kamali-Sarvestani 2007 & 68 & 406 & 20 & 224 & $3.7 \%$ & $2.05[1.21,3.48]$ & 2007 & & & $\rightarrow$ & \\
\hline Beghe 2009 & 72 & 598 & 42 & 352 & $4.3 \%$ & $1.01[0.67,1.52]$ & 2009 & & & - & \\
\hline Rad 2010 & 18 & 128 & 23 & 130 & $3.1 \%$ & $0.76[0.39,1.49]$ & 2010 & & & - & \\
\hline Baye-1 2011 & 161 & 826 & 69 & 596 & $4.8 \%$ & $1.85[1.36,2.51]$ & 2011 & & & $\rightarrow$ & \\
\hline Baye-2 2011 & 209 & 630 & 48 & 102 & $4.3 \%$ & $0.56[0.37,0.85]$ & 2011 & & , & & \\
\hline Total $(95 \% \mathrm{Cl})$ & & 9204 & & 6962 & $100.0 \%$ & $1.20[1.01,1.42]$ & & & & 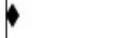 & \\
\hline Total events & 4675 & & 3356 & & & & & & & & \\
\hline \multicolumn{8}{|c|}{$\begin{array}{l}\text { Heterogeneity: } \operatorname{Tau}^{2}=0.14 ; \mathrm{Chi}^{2}=98.72, \mathrm{df}=23(\mathrm{P}<0.00001) ; \mathrm{I}^{2}=77 \% \\
\text { Test for overall effect: } Z=2.04(P=0.04)\end{array}$} & 0.01 & $\begin{array}{l}0.1 \\
\text { control }\end{array}$ & $1 \underset{10}{10}$ & 100 \\
\hline
\end{tabular}

Figure 1. Odds ratio and $95 \%$ confidence interval $(95 \% \mathrm{CI})$ from individual studies testing association of the C-589T polymorphisms and asthma.

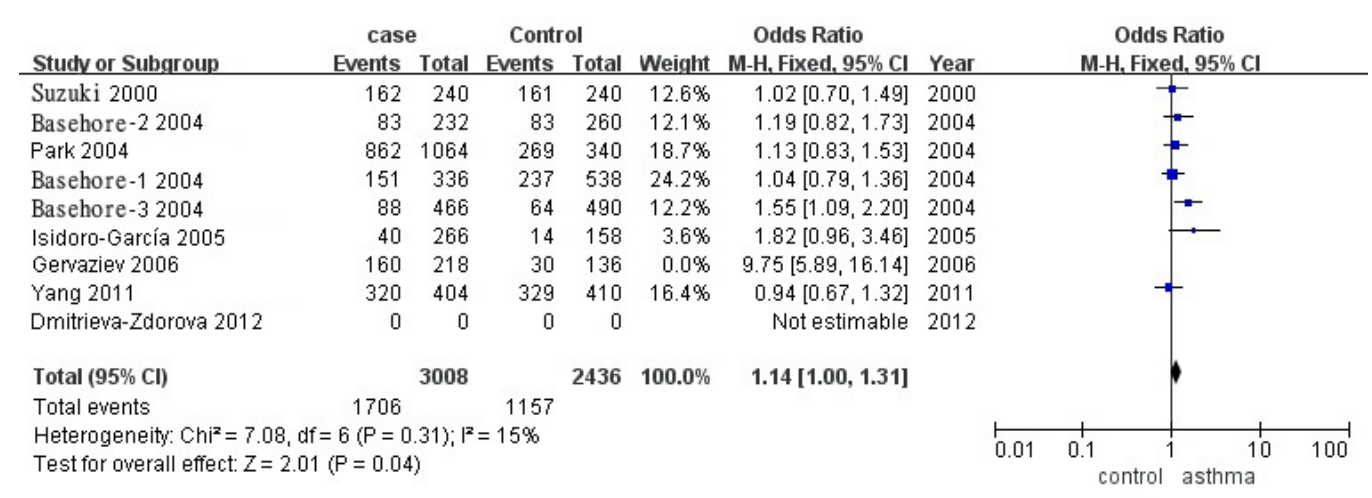

Figure 2. Odds ratio and $95 \%$ confidence interval $(95 \% \mathrm{CI})$ from individual studies testing association of the C-33T polymorphisms and asthma.

No significant association was found between C-589T and asthma when stratified by age, except for the $\mathrm{TT}+\mathrm{CT}$ (dominant) genotype in adults. This result was in agreement with the overall results of the genotype analysis (Figure 5). 


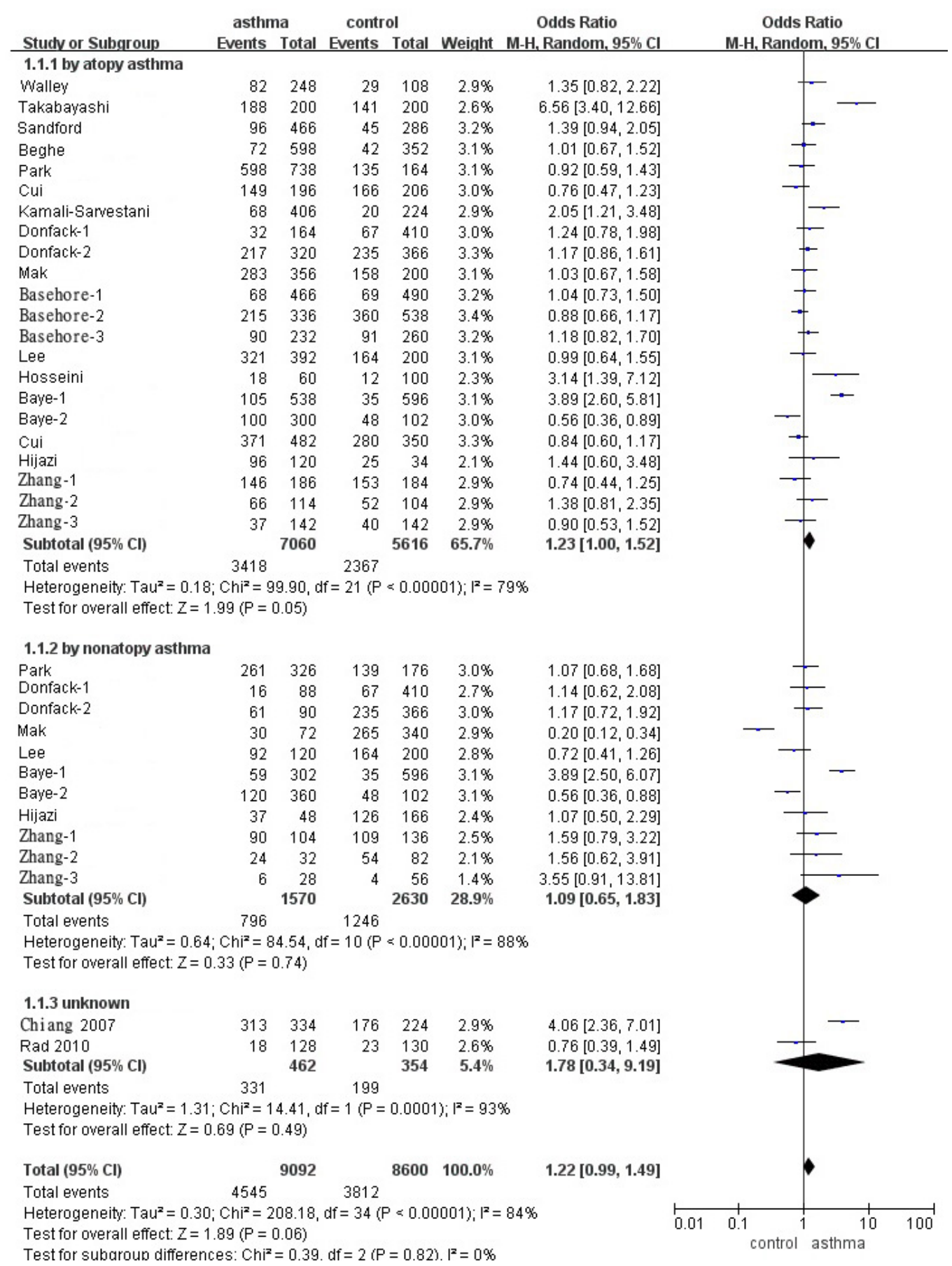

Figure 3. Forest plot of asthma risk associated with the C-589T polymorphism in the stratified analyses by asthma status. 


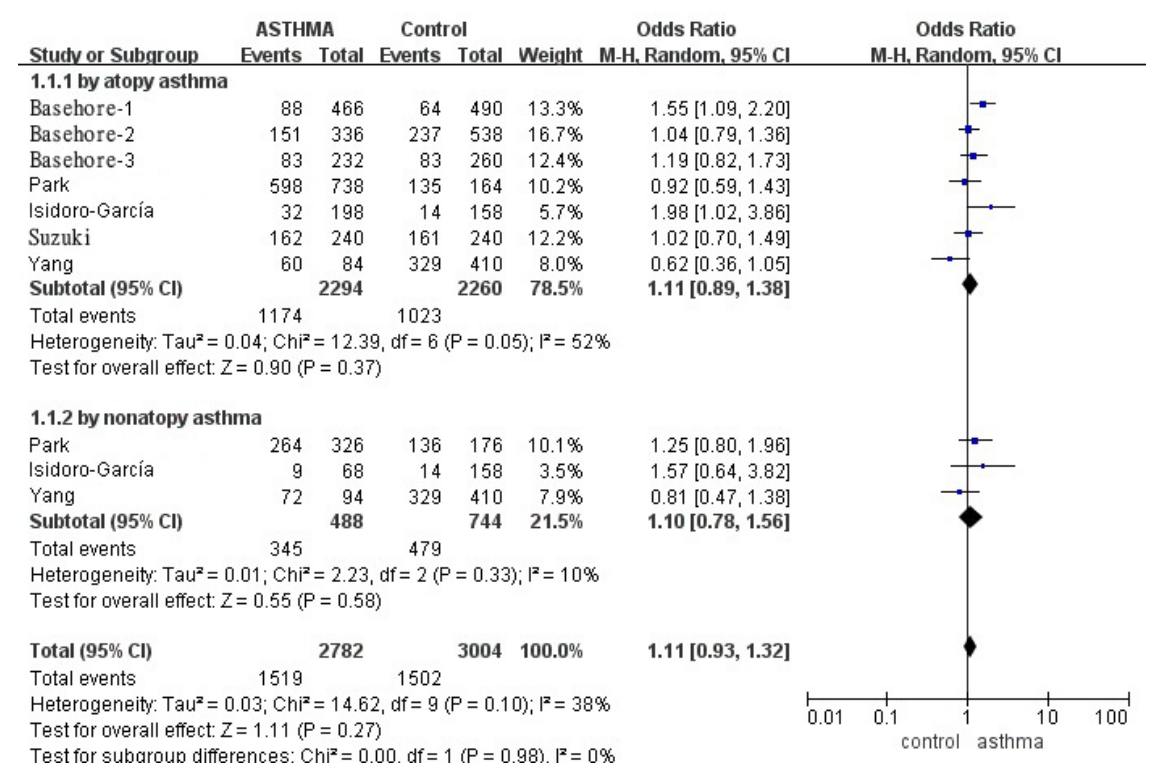

Figure 4. Forest plot of asthma risk associated with the C-33T polymorphism in the stratified analyses by asthma status.

\begin{tabular}{|c|c|c|c|c|c|c|c|c|c|c|c|}
\hline Studv or Subgroup & \multicolumn{2}{|c|}{ case } & \multicolumn{2}{|c|}{ Control } & \multicolumn{3}{|c|}{ Odds Ratio } & \multicolumn{4}{|c|}{$\begin{array}{l}\text { Odds Ratio } \\
\text { M-H, Random, } 95 \% \mathrm{Cl}\end{array}$} \\
\hline \multicolumn{12}{|l|}{ 1.1.1 by adult } \\
\hline Walley 1996 & 82 & 248 & 29 & 108 & $3.9 \%$ & $1.35[0.82,2.22]$ & 1996 & & & - & \\
\hline Sandford 2000 & 96 & 466 & 45 & 286 & $4.4 \%$ & $1.39[0.94,2.05]$ & 2000 & & & - & \\
\hline Hijazi 2000 & 133 & 168 & 151 & 200 & $3.9 \%$ & $1.23[0.75,2.02]$ & 2000 & & & & \\
\hline Cui 2003 & 371 & 482 & 280 & 350 & $4.7 \%$ & $0.84[0.60,1.17]$ & 2003 & & & & \\
\hline Cui 2003 & 149 & 196 & 166 & 206 & $4.0 \%$ & $0.76[0.47,1.23]$ & 2003 & & & & \\
\hline Park 2004 & 862 & 1064 & 272 & 340 & $4.8 \%$ & $1.07[0.79,1.45]$ & 2004 & & & & \\
\hline Basehore-1 2004 & 68 & 466 & 69 & 490 & $4.6 \%$ & $1.04[0.73,1.50]$ & 2004 & & & & \\
\hline Basehore-2 2004 & 215 & 336 & 360 & 538 & $4.9 \%$ & $0.88[0.66,1.17]$ & 2004 & & & & \\
\hline Basehore-3 2004 & 90 & 232 & 91 & 260 & $4.5 \%$ & $1.18[0.82,1.70]$ & 2004 & & & & \\
\hline Zhang-1 2005 & 236 & 290 & 262 & 312 & $4.3 \%$ & $0.83[0.55,1.27]$ & 2005 & & & & \\
\hline Zhang-3 2005 & 43 & 170 & 44 & 198 & $4.0 \%$ & $1.19[0.73,1.92]$ & 2005 & & & & \\
\hline Donfack-2 2005 & 278 & 410 & 235 & 366 & $4.9 \%$ & $1.17[0.87,1.58]$ & 2005 & & & & \\
\hline Zhang-2 2005 & 90 & 146 & 116 & 186 & $4.1 \%$ & $0.97[0.62,1.52]$ & 2005 & & & & \\
\hline Donfack-1 2005 & 49 & 252 & 81 & 410 & $4.4 \%$ & $0.98[0.66,1.46]$ & 2005 & & & & \\
\hline Hosseini 2007 & 18 & 60 & 12 & 100 & $2.5 \%$ & $3.14[1.39,7.12]$ & 2007 & & & & \\
\hline Mak 2007 & 453 & 584 & 459 & 584 & $5.0 \%$ & $0.94[0.71,1.24]$ & 2007 & & & & \\
\hline Kamali-Sarvestani 2007 & 68 & 406 & 20 & 224 & $3.7 \%$ & $2.05[1.21,3.48]$ & 2007 & & & & \\
\hline Chiang 2007 & 313 & 334 & 176 & 224 & $3.6 \%$ & $4.06[2.36,7.01]$ & 2007 & & & $\rightarrow$ & \\
\hline Beghe 2009 & 72 & 598 & 42 & 352 & $4.3 \%$ & $1.01[0.67,1.52]$ & 2009 & & & & \\
\hline $\operatorname{Rad} 2010$ & 18 & 128 & 23 & 130 & $3.1 \%$ & $0.76[0.39,1.49]$ & 2010 & & & & \\
\hline Subtotal $(95 \% \mathrm{Cl})$ & & 7036 & & 5864 & $83.5 \%$ & $1.14[0.98,1.32]$ & & & & & \\
\hline Total events & 3704 & & 2933 & & & & & & & & \\
\hline \multicolumn{12}{|c|}{ 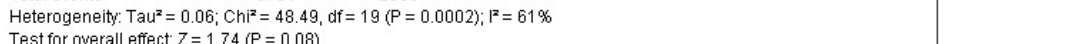 } \\
\hline \multicolumn{12}{|c|}{ Test for overall effect: $Z=1.74(P=0.08)$} \\
\hline \multicolumn{12}{|l|}{ 1.1.2 by child } \\
\hline Takabayashi 2000 & 188 & 200 & 141 & 200 & $3.1 \%$ & $6.56[3.40,12.66]$ & 2000 & & & - & \\
\hline Lee 2004 & 413 & 512 & 165 & 200 & $4.2 \%$ & $0.88[0.58,1.35]$ & 2004 & & & & \\
\hline Baye-2 2011 & 209 & 630 & 48 & 102 & $4.3 \%$ & $0.56[0.37,0.85]$ & 2011 & & $\rightarrow$ & & \\
\hline Baye-1 2011 & 161 & 826 & 69 & 596 & $4.8 \%$ & $1.85[1.36,2.51]$ & 2011 & & & & \\
\hline Subtotal $(95 \% \mathrm{Cl})$ & & 2168 & & 1098 & $16.5 \%$ & $1.52[0.65,3.54]$ & & & & & \\
\hline Total events & 971 & & 423 & & & & & & & & \\
\hline \multirow{2}{*}{\multicolumn{12}{|c|}{$\begin{array}{l}\text { Heterogeneity: } \operatorname{Tau}^{2}=0.69 ; C h i^{2}=47.08, d f=3(P<0.00001) ; I^{2}=94 \% \\
\text { Test for overall effect: } Z=0.97(P=0.33)\end{array}$}} \\
\hline & & & & & & & & & & & \\
\hline Total $(95 \% \mathrm{Cl})$ & & 9204 & & 6962 & $100.0 \%$ & $1.20[1.01,1.42]$ & & & & $b$ & \\
\hline Total events & 4675 & & 3356 & & & & & & & & \\
\hline Heterogeneity: $\operatorname{Tau}^{2}=0$ & $\mathrm{Chi}^{2}=98$ & $72, \mathrm{df}=$ & $23(P<0$ & 0.00001 & ); $1^{2}=77 \%$ & & & & & 10 & 100 \\
\hline Test for overall effect: $Z$ & $4(P=0.0$ & & & & & & & & control & asthma & \\
\hline
\end{tabular}

Figure 5. Forest plot of asthma risk associated with the C-589T polymorphism in the stratified analyses by age. 


\section{Heterogeneity and publication bias}

There was heterogeneity for C-589T, the T allele, and genotype in the meta-analysis. Significant heterogeneity was found with respect to Asian origin, asthma status, and age. Sensitivity analysis excluding individual studies was performed if the meta-analysis achieved similar results, which indicated the robustness of the meta-analysis. Furthermore, the Egger test revealed no evidence of publication bias (Table 2).

\section{DISCUSSION}

In the present study, we conducted a meta-analysis to assess the genetic association between the IL-4 promoters C-589T, C-33T, and G-1098T polymorphisms and asthma. The results of this meta-analysis provide strong evidence of association between C-589T and C$33 \mathrm{~T}$ with asthma in certain populations.

IL-4 is an important factor in the pathogenesis of allergies, which has been verified using animal models (Pessi et al., 2005). IL-4 has clearly been shown to play a crucial role in the pathogenesis of allergic diseases including asthma, and IL-4 increases airway responsiveness by recruiting eosinophils into the airway in patients with allergic asthma (Dizier et al., 1999).

It is reasonable to infer that scaling expression of IL-4 might lead to atopic diseases. Therefore, it is highly possible that polymorphisms of the IL-4 promoter region would most likely contribute to its increasing expression. A large number of SNPs of IL-4 have been identified, which have shown to be significantly correlated with disease risks. For example, two general SNPs have been implicated in allergic diseases (including asthma) in many studies. rs2243250 $(-589 \mathrm{C} / \mathrm{T})$ was reported to be associated with increased binding of a nuclear transcription factor. The other SNP (-33C/T; rs2070874) was located in the 5'-UTR. Both SNPs were found to be in linkage disequilibrium in European and Asian populations (Donfack et al., 2005; Yang et al., 2011).

In the present meta-analysis, we investigated 21 studies (6421 cases and 4755 controls) to assess the association of IL-4 gene promoter polymorphisms with asthma. Compared with a previous meta-analysis by $\mathrm{Li}$ et al. (2008), we found a significant association of IL-4 promoter polymorphisms (C-589T and C-33T) with asthma. The IL-4-589T allele has been shown to be associated with pathogenesis of asthma in populations and family-based cohorts (Noguchi et al., 1998; Beghe et al., 2003). Genetic heterogeneity or statistical power may contribute to the different results of case-control and family-based studies. For C-589T, the $\mathrm{TT}+\mathrm{CT}$ genotype, and not the TT genotype, was significantly associated with asthma. Although the dominant model $(\mathrm{CC}+\mathrm{CT} / \mathrm{TT})$ was not associated with asthma $(\mathrm{OR}=0.90,95 \% \mathrm{CI}$ $=0.69-1.184, \mathrm{P}=0.44)$, the recessive model $(\mathrm{CC} / \mathrm{CT}+\mathrm{TT})$ was significantly associated with asthma $(\mathrm{OR}=1.30,95 \% \mathrm{CI}=1.11-1.53, \mathrm{P}=0.001)$. This result may suggest a recessive effect of the $\mathrm{C}$ allele. However, for C-33T, no significant association was found between genotypes and asthma, which may be due to the limitation of the available studies.

The association between IL-4 C-589T and asthma was only found among individuals of European origin, and not in individuals of Asian or African-American origin $(\mathrm{OR}=1.31$, $95 \% \mathrm{CI}=0.99-1.74, \mathrm{P}=0.06$ and $\mathrm{OR}=0.85,95 \% \mathrm{CI}=0.58-1.25, \mathrm{P}=0.42$, respectively). Ethnic differences may not affect the pathogenesis of asthma in different populations. Furthermore, the discrepancy of individual studies may be due to small sample sizes, poor statistical power (power $<80$ ), or clinical heterogeneity. 
Results of the association between C-33T and asthma were slightly different. The association between IL-4 C-33T and asthma was identified in the European origin group but not in the Asian origin group. Genotype analysis revealed no significant association between C-33T and asthma, although we could not elucidate the genetic effect on the pathogenesis of asthma due to insufficiency of studies. The contradictory results among the European origin, Asian origin, and African-American origin groups might indicate genetic heterogeneity of asthma among these three populations.

Results based on asthma status and age differed from the combined results, which might imply that asthma status and age may not be contributing factors on the pathogenesis of asthma. To clarify the association between IL-4 promoter SNPs and asthma, more research with more SNPs needs to be considered.

Heterogeneity was detected both in the allele and genotype analyses, while the sensitivity analysis and publication bias analysis demonstrated the authenticity of this study. Since no significant associations were detected between the C-589T and C-33T polymorphisms and asthma in most published studies reported, publication bias is likely not a major problem for this meta-analysis.

Nevertheless, several limitations of this meta-analysis should be considered. First, considering the association between C-589T and asthma, heterogeneity was discovered in the Asian origin group. However, based on the sensitivity analysis, the overall effect was not influenced by heterogeneity. Second, the number of studies and subjects included in this meta-analysis were relatively small. There were less than three studies included for the polymorphism meta-analysis in subgroups. Therefore, more studies are needed for further analysis. Third, due to lack of sufficient studies, we eliminated the meta-analysis of G-1098T, and it is possible that several other variants in the IL-4 promoter may be associated with asthma susceptibility. Therefore, further research is required.

In conclusion, this meta-analysis, which included 13,536 subjects, demonstrated that IL-4 promoter polymorphisms were associated with asthma susceptibility only in European groups. To identify associations between polymorphisms in IL-4 promoter SNPs and asthma susceptibility, more studies with a larger number of patients are needed.

\section{ACKNOWLEDGMENTS}

Research supported by the Education Department Project of Hunnan Province (grant $\# 12 \mathrm{C} 0523)$.

\section{REFERENCES}

Amirzargar AA, Movahedi M, Rezaei N, Moradi B, et al. (2009). Polymorphisms in IL4 and IL4RA confer susceptibility to asthma. J. Invest. Allergol. Clin. Immunol. 19: 433-438.

Basehore MJ, Howard TD, Lange LA, Moore WC, et al. (2004). A comprehensive evaluation of IL4 variants in ethnically diverse populations: association of total serum IgE levels and asthma in white subjects. J. Allergy Clin. Immunol. 114: 80-87.

Baye TM, Butsch KM, Biagini Myers JM, Martin LJ, et al. (2011). Differences in candidate gene association between European ancestry and African American asthmatic children. PLoS One 6: e16522.

Beghe B, Barton S, Rorke S, Peng Q, et al. (2003). Polymorphisms in the interleukin-4 and interleukin-4 receptor alpha chain genes confer susceptibility to asthma and atopy in a Caucasian population. Clin. Exp. Allergy 33: 1111-1117.

Beghe B, Hall IP, Parker SG, Moffatt MF, et al. (2010). Polymorphisms in IL13 pathway genes in asthma and chronic 
obstructive pulmonary disease. Allergy 65: 474-481.

Berenguer AG, Camara RA, Brehm AD, Oliveira S, et al. (2012). Distribution of polymorphisms IL4-590 C/T and IL4 RP2 in the human populations of Madeira, Azores, Portugal, Cape Verde and Guinea-Bissau. Int. J. Mol. Epidemiol. Genet. 3: 179-183.

Chiang CH, Tang YC, Lin MW and Chung MY (2007). Association between the IL-4 promoter polymorphisms and asthma or severity of hyperresponsiveness in Taiwanese. Respirology 12: 42-48.

Cui TP, Wang L and Wu JM (2003a). Polymorphisms in the IL-4 and IL-4R genes and allergic asthma in adult. Immunol. J. 19: 454-459.

Cui TP, Wu J, Pan S and Xie J (2003b). Polymorphisms in the IL-4 and IL-4R [alpha] genes and allergic asthma. Clin. Chem. Lab. Med. 41: 888-892.

Dizier MH, Sandford A, Walley A, Philippi A, et al. (1999). Indication of linkage of serum IgE levels to the interleukin-4 gene and exclusion of the contribution of the (-590 C to T) interleukin-4 promoter polymorphism to IgE variation. Genet. Epidemiol. 16: 84-94.

Dmitrieva-Zdorova EV, Voronko OE, Latysheva EA, Storozhakov GI, et al. (2012). Analysis of polymorphisms in T(H)2associated genes in Russian patients with atopic bronchial asthma. J. Investig. Allergol. Clin. Immunol. 22: 126-132.

Donfack J, Schneider DH, Tan Z, Kurz T, et al. (2005). Variation in conserved non-coding sequences on chromosome 5q and susceptibility to asthma and atopy. Respir. Res. 6: 145.

Elliott K, Fitzpatrick E, Hill D, Brown J, et al. (2001). The -590C/T and -34C/T interleukin-4 promoter polymorphisms are not associated with atopic eczema in childhood. J. Allergy Clin. Immunol. 108: 285-287.

Freidin MB, Kobyakova OS, Ogorodova LM and Puzyrev VP (2003). Association of polymorphisms in the human IL4 and IL5 genes with atopic bronchial asthma and severity of the disease. Comp. Funct. Genomics 4: 346-350.

Gervaziev YV, Kaznacheev VA and Gervazieva VB (2006). Allelic polymorphisms in the interleukin-4 promoter regions and their association with bronchial asthma among the Russian population. Int. Arch. Allergy Immunol. 141: 257-264.

Haller G, Torgerson DG, Ober C and Thompson EE (2009). Sequencing the IL4 locus in African Americans implicates rare noncoding variants in asthma susceptibility. J. Allergy Clin. Immunol. 124: 1204-1209.

Hijazi Z and Haider MZ (2000). Interleukin-4 gene promoter polymorphism [C590T] and asthma in Kuwaiti Arabs. Int. Arch. Allergy Immunol. 122: 190-194.

Hosseini-Farahabadi S, Tavakkol-Afshari J, Rafatpanah H, Farid-Hosseini R, et al. (2007). Association between the polymorphisms of IL-4 gene promoter (-590C/T), IL-13 coding region (R130Q) and IL-16 gene promoter (-295T/C) and allergic asthma. Iran J. Allergy Asthma Immunol. 6: 9-14.

Isidoro-García M, Dávila I, Laffond E, Moreno E, et al. (2005). Interleukin-4 (IL4) and Interleukin-4 receptor (IL4RA) polymorphisms in asthma: a case control study. Clin. Mol. Allergy 3: 15.

Kamali-Sarvestani E, Ghayomi MA and Nekoee A (2007). Association of TNF- $\alpha-308$ G/A and IL-4 -589 C/T gene promoter polymorphisms with asthma susceptibility in the south of Iran. J. Investig. Allergol. Clin. Immunol. 17: 361-366.

Kim BS, Park SM, Uhm TG, Kang JH, et al. (2010). Effect of single nucleotide polymorphisms within the interleukin-4 promoter on aspirin intolerance in asthmatics and interleukin-4 promoter activity. Pharmacogenet. Genomics 20: 748-758.

Laitinen T, Kauppi P, Ignatius J, Ruotsalainen T, et al. (1997). Genetic control of serum IgE levels and asthma: linkage and linkage disequilibrium studies in an isolated population. Hum. Mol. Genet. 6: 2069-2076.

Lee SG, Kim BS, Kim JH, Lee SY, et al. (2004). Gene-gene interaction between interleukin-4 and interleukin-4 receptor alpha in Korean children with asthma. Clin. Exp. Allergy 34: 1202-1208.

Li Y, Guo B, Zhang L, Han J, et al. (2008). Association between C-589T polymorphisms of interleukin-4 gene promoter and asthma: a meta-analysis. Respir. Med. 102: 984-992.

Liu X, Beaty TH, Deindl P, Huang SK, et al. (2003). Associations between total serum IgE levels and the 6 potentially functional variants within the genes IL4, IL13, and IL4RA in German children: the German Multicenter Atopy Study. J. Allergy Clin. Immunol. 112: 382-388.

Mak JC, Ko FW, Chu CM, Leung HC, et al. (2007). Polymorphisms in the IL-4, IL-4 receptor alpha chain, TNF-alpha, and lymphotoxin-alpha genes and risk of asthma in Hong Kong Chinese adults. Int. Arch. Allergy Immunol. 144: 114-122.

Michel S, Liang L, Depner M, Klopp N, et al. (2010). Unifying candidate gene and GWAS approaches in asthma. PLoS One 5: e13894.

Noguchi E, Shibasaki M, Arinami T, Takeda K, et al. (1998). Association of asthma and the interleukin-4 promoter gene in Japanese. Clin. Exp. Allergy 28: 449-453.

Noguchi E, Nukaga-Nishio Y, Jian Z, Yokouchi Y, et al. (2001). Haplotypes of the 5' region of the IL-4 gene and SNPs in the intergene sequence between the IL-4 and IL-13 genes are associated with atopic asthma. Hum. Immunol. 62: 
1251-1257.

Park BL, Kim LH, Choi YH, Lee JH, et al. (2004). Interleukin 3 (IL3) polymorphisms associated with decreased risk of asthma and atopy. J. Hum. Genet. 49: 517-527.

Pessi T, Virta M, Adjers K, Karjalainen J, et al. (2005). Genetic and environmental factors in the immunopathogenesis of atopy: interaction of Helicobacter pylori infection and IL4 genetics. Int. Arch. Allergy Immunol. 137: 282-288.

Rad IA, Bagheri M, Rahimi-Rad MH and Moradi Z (2010). IFN- $\gamma+874$ and IL-4 -590 polymorphisms and asthma susceptibility in North West of Iran. Tanaffos 9: 22-27.

Renauld JC (2001). New insights into the role of cytokines in asthma. J. Clin. Pathol. 54: 577-589.

Rosenwasser LJ, Klemm DJ, Dresback JK, Inamura H, et al. (1995). Promoter polymorphisms in the chromosome 5 gene cluster in asthma and atopy. Clin. Exp. Allergy 25 (Suppl 2): 74-78.

Sandford AJ, Chagani T, Zhu S, Weir TD, et al. (2000). Polymorphisms in the IL4, IL4RA, and FCERIB genes and asthma severity. J. Allergy Clin. Immunol. 106: 135-140.

Suzuki I, Hizawa N, Yamaguchi E and Kawakami Y (2000). Association between a C+33T polymorphism in the IL-4 promoter region and total serum IgE levels. Clin. Exp. Allergy 30: 1746-1749.

Takabayashi A, Ihara K, Sasaki Y, Suzuki Y, et al. (2000). Childhood atopic asthma: positive association with a polymorphism of IL-4 receptor alpha gene but not with that of IL-4 promoter or Fc epsilon receptor I $\beta$ gene. Exp. Clin. Immunogenet. 17: 63-70.

Walley AJ and Cookson WO (1996). Investigation of an interleukin-4 promoter polymorphism for associations with asthma and atopy. J. Med. Genet. 33: 689-692.

Wang XH, Zhao W, Liu SG and Feng XP (2009). Correlation of IL-4 and IL-13 gene polymorphisms with asthma and total serum IgE levels. Zhonghua Jie. He. He. Hu Xi. Za Zhi. 32: 161-164.

Yang XX, Li FX, Wu YS, Wu D, et al. (2011). Association of TGF- $\beta 1$, IL-4 and IL-13 gene polymerphisms with asthma in a Chinese population. Asian Pac. J. Allergy Immunol. 29: 273-277.

Zhang WD, Zhang XZ and Qiu DW (2005). Association of interleukin-4 and interleukin-4 receptor gene polymorphism and serum IgE levels in Chinese, Malayan and Hindoo. Zhonghua Jie He He Hu Xi Za Zhi. 28: 489-490.

Zhu S, Chan-Yeung M, Becker AB, Dimich-Ward H, et al. (2000). Polymorphisms of the IL-4, TNF- $\alpha$, and Fc $\alpha$-RI $\beta$ genes and the risk of allergic disorders in at-risk infants. Am. J. Respir. Crit. Care Med. 161: 1655-1659. 